\title{
Strong vector variational like inequality problems with properly quasimonotone bifunctions
}

Monika Mehta and Mamta Chaudhary*

\section{"Correspondence:}

mam.gupta18@gmail.com

Department of Mathematics,

Satyawati College, University of

Delhi, Ashok Vihar, Delhi, 110052, India

\begin{abstract}
In this paper, we introduce two types of proper quasimonotone maps over cones for a vector-valued bifunction and discuss their relations with generalized monotone maps, namely cone pseudomonotone and cone quasimonotone maps. Strong vector variational like inequality problems of the Stampacchia and the Minty type have been defined. These problems are a generalization of the classical Stampacchia and Minty problems and encompass many problems studied in the literature. A generalization of celebrated Minty lemma, relating the solutions of the two problems, has been proved. Existence results for strong Stampacchia and Minty type vector variational like inequality problems have been established using the notions of proper quasimonotone maps over cones. Gap functions have also been proposed for both problems.
\end{abstract}

Keywords: vector variational like inequalities; bifunctions; proper cone-quasimonotonicity; cone-quasimonotonicity; cone-pseudomonotonicity

\section{Introduction}

The study of vector variational inequality problem in finite dimensional spaces was initiated by Giannessi [1]. Vector variational inequalities have proved to be efficient tools for investigating vector optimization problems and they also provide with a mathematical model for the problems of equilibrium in a mechanical structure when there are several aspects (weight, cost, resistance etc.) which are in conflict. Because of these applications, the study of vector variational inequalities has attracted wide attention.

A useful and important generalization of the vector variational inequality problem is the vector variational like inequality problem, which has been studied and investigated by many authors like Ansari [2], Garzon et al. [3], Al-Homidan, Ansari and Yao [4], Lee and Lee [5], Li and He [6].

The classical Stampacchia variational inequality problem is to find a vector $x \in K$ such that

$$
\langle T x, y-x\rangle \geq 0, \quad \forall y \in K,
$$

where $K \subseteq R^{n}$ is a nonempty closed convex set, $T: R^{n} \rightarrow R^{n}$ and the Minty variational inequality problem is to find a vector $x \in K$ such that

$$
\langle T y, x-y\rangle \leq 0, \quad \forall y \in K
$$

O2014 Mehta and Chaudhary; licensee Springer. This is an Open Access article distributed under the terms of the Creative Commons Attribution License (http://creativecommons.org/licenses/by/2.0), which permits unrestricted use, distribution, and reproduction in any medium, provided the original work is properly cited. 
It is well known that in contrast to the Stampacchia problem, compactness and convexity of $K$ and continuity assumptions on $T$ do not guarantee the existence of a solution to (MVI) and that some generalized monotonicity assumptions on $T$ are needed. Proper quasimonotonicity, as introduced by Daniilidis and Hadjisavvas [7], plays a significant role in establishing existence results for (MVI) (see [8]).

A generalization and an extension of the problems (SVI) and (MVI) to the vector case was studied by Lalitha and Mehta in $[9,10]$ where a general vector bifunction $h: K \times R^{n} \rightarrow$ $\bar{R}^{m}$ was considered, where $\bar{R}=R \cup\{+\infty,-\infty\}$ and $K \subseteq R^{n}$ is a nonempty closed convex subset. A systematic study of the problem for the scalar case can be found in Ansari et al. [11]. The Stampacchia type vector variational inequality problem (SVVI) considered in [9] was to find $x \in K$ such that

$$
h(x ; y-x) \notin-\operatorname{int} C, \quad \forall y \in K,
$$

and the Minty type vector variational inequality problem (MVVI) considered was to find $x \in K$ such that

$$
h(y ; x-y) \notin-\operatorname{int} C, \quad \forall y \in K,
$$

where $C \subseteq R^{m}$ is a pointed closed convex cone with nonempty interior. In [10] the problem (SVVI) was studied with a varying cone $C(x)$.

The role of monotonocity in variational inequality theory is the same as that of convexity in optimization theory. In recent years, a number of authors have used many important generalizations of monotonicity such as quasimonotonicity, pseudomonotonicity, dense pseudomonotonicity, $p$-monotonicity, semimonotonicity, and they have considered these notions to study various variational inequalities and other related problems [7, 9, 12-17]. In this paper, we extend the notion of proper quasimonotonicity to vector-valued bifunctions and use it to establish the existence of solutions to strong Stampacchia and Minty type vector variational like inequalities problems defined by a bifunction $h$.

The paper is organized as follows. In Section 2, the notions of cone proper quasimonotonicity of Stampacchia and Minty type for a vector-valued bifunction are introduced. Their relations with the class of cone pseudomonotone and cone quasimonotone maps are discussed in this section. In Section 3 two types of strong vector variational like inequality problems, of Stampacchia and Minty kind, are introduced in terms of a general bifunction. Conditions which ensure the equivalence of these problems and existence of solutions for both problems form the basis of this section. In the last section two types of gap functions are proposed for each of the problems. One of the gap functions is given for the case where the cone considered is the nonnegative orthant.

\section{Proper cone quasimonotonicity for vector-valued bifunctions}

This section has been divided into two subsection. In the first subsection we introduce two types of proper quasimonotone maps over cones for a vector-valued bifunction $h$ and give a set of sufficient conditions for such maps. The second subsection investigates the relation of proper quasimonotonocity introduced in the previous subsection with other generalized monotonicity. 
Throughout this paper, we assume the vector-valued bifunctions $h: K \times R^{n} \rightarrow \bar{R}^{m}$ and $\eta: K \times K \rightarrow R^{n}$ to be defined on a nonempty closed convex subset $K$ of $R^{n}, C$ to be a closed convex pointed cone in $R^{m}$ with nonempty interior.

\subsection{Stampacchia and Minty type proper quasimonotonicity}

Definition 2.1 The bifunction $h$ is said to be

(i) $(C, \eta)$-pseudomonotone on $K$ if for any $x, y \in K h(x ; \eta(y, x)) \in C \Rightarrow h(y ; \eta(x, y)) \in-C$;

(ii) $(C, \eta)$-quasimonotone on $K$ if for any $x, y \in K h(x ; \eta(y, x)) \in$ int $C \Rightarrow h(y ; \eta(x, y)) \in-C$.

The above definition of quasimonotonicity appears for a general vector-valued bifunction $F: K \times K \rightarrow R^{n}$ in [18]. When $h(x ; \eta(y, x))=\langle T(x), y-x\rangle$ where $T: R^{n} \rightarrow R^{m \times n}$ and $\eta(x, y)=x-y$ then the definition of $(C, \eta)$-pseudomonotonicity reduces to the one considered by Komlosi in [15] referred to as neutral $C$-pseudomonotonicity.

From the above definition it is clear that every $(C, \eta)$-pseudomonotone bifunction is $(C, \eta)$-quasimonotone but the converse implication is not necessarily true which is illustrated by the following example.

Example 2.1 Let $K=[0, \pi]$ and $h: K \times R \rightarrow \bar{R}^{2}$ be defined as $h(x ; d)=\left(d \sin ^{2} x, d^{3} x^{2}\right)$. Let $C=R_{+}^{2}$ and $\eta: K \times K \rightarrow R$ be given as $\eta(x, y)=\cos x-\cos y$. Then it can easily be verified that $h$ is $(C, \eta)$-quasimonotone on $K$, but not $(C, \eta)$-pseudomonotone on $K$ because for $x=0, y=\pi / 2, h(x ; \eta(y, x))=(0,0) \in C$ but $h(y ; \eta(x, y))=\left(1, \pi^{2} / 4\right) \notin-C$.

The notion of proper quasimonotonicity for an operator was introduced by Daniilidis and Hadjisavvas in [7]. An earlier version under the name of 0-diagonal quasiconcavity was introduced in [19]. The concept of proper quasimonotonicity for an extended realvalued bifunction $h: K \times R \rightarrow \bar{R}$ has been given by Bianchi and Pini [20] as follows. The bifunction $h$ is said to be properly quasimonotone if for every $y_{1}, y_{2}, \ldots, y_{p} \in K$ and $x \in$ $\operatorname{co}\left\{y_{1}, y_{2}, \ldots, y_{p}\right\}$ there exists $i \in\{1,2, \ldots, p\}$ such that $h\left(y_{i} ; x-y_{i}\right) \leq 0$, where $\operatorname{co}\left\{y_{1}, y_{2}, \ldots, y_{p}\right\}$ denotes the convex hull of $\left\{y_{1}, y_{2}, \ldots, y_{p}\right\}$.

We now extend the above definition to the vector case in terms of $\eta$.

Definition 2.2 If for every $y_{1}, y_{2}, \ldots, y_{p} \in K$ and $x \in \operatorname{co}\left\{y_{1}, y_{2}, \ldots, y_{p}\right\}$ there exists $i \in$ $\{1,2, \ldots, p\}$ such that

(i) $h\left(x ; \eta\left(y_{i}, x\right)\right) \in C$, then $h$ is said to be properly $(C, \eta)$-quasimonotone of the Stampacchia type on $K$;

(ii) $h\left(y_{i} ; \eta\left(x, y_{i}\right)\right) \in-C$, then $h$ is said to be properly $(C, \eta)$-quasimonotone of the Minty type on $K$.

\section{Remark 2.1}

(i) If $m=1, C=R_{+}$and $\eta(x, y)=x-y$ then the definition of proper

$(C, \eta)$-quasimonotonicity of the Minty type reduces to the definition of proper quasimonotonicity given by Bianchi and Pini [20].

(ii) If $h(x ; \eta(y, x))=\langle T x, \eta(y, x)\rangle$, where $T: K \rightarrow L\left(R^{n}, R^{m}\right), L\left(R^{n}, R^{m}\right)$ is the space of all continuous linear mappings from $R^{n}$ to $R^{m}$, that is, for any $l \in L\left(R^{n}, R^{m}\right)$ and $x \in R^{n}$, $\langle l, x\rangle$ denotes the value of $l$ at $x$, then the above definition is the finite dimensional version of Definition 2.5 given by Zhao and Xia [16]. 
Remark 2.2 The bifunction $h$ considered in Example 2.1 is properly $(C, \eta)$-quasimonotone on $K$ of the Stampacchia type. Suppose on the contrary there exist $y_{1}, y_{2}, \ldots, y_{p} \in K$ and $\lambda_{i} \geq 0, i=1,2, \ldots, p$ with $\sum_{i=1}^{p} \lambda_{i}=1$ and $x=\sum_{i=1}^{p} \lambda_{i} y_{i}$ such that $h\left(x ; \eta\left(y_{i}, x\right)\right) \notin C, \forall i=$ $1,2, \ldots, p$, which implies that $x>0$ and $\cos y_{i}<\cos x$. The second inequality implies that $y_{i}>x, \forall i=1,2, \ldots, p$. Summing over $i$ we have $x>x$, which is a contradiction. Similarly it can be established that $h$ is also properly $(C, \eta)$-quasimonotone of the Minty type on $K$.

The following two examples elucidate that the two classes of properly $(C, \eta)$-quasimonotonicity are independent in the sense that a bifunction may be properly quasimonotone of the Stampacchia type but may not be properly quasimonotone of the Minty type and vice versa.

Example 2.2 Let $K=R_{+}$and $h: K \times R \rightarrow \bar{R}^{2}$ be defined as $h(x ; d)=\left(x^{2} d, x d^{5}\right)$. Let $C=R_{+}^{2}$, and let $\eta: K \times K \rightarrow R$ be defined as $\eta(x, y)=x-y+y^{4}$. We assert that $h$ is properly $(C, \eta)$-quasimonotone of the Stampacchia type on $K$. Suppose on the contrary there exist $y_{1}, y_{2}, \ldots, y_{p} \in K$ and $\lambda_{i} \geq 0, i=1,2, \ldots, p$ with $\sum_{i=-1}^{p} \lambda_{i}=1$ and $x=\sum_{i=1}^{p} \lambda_{i} y_{i}$ such that $h\left(x ; \eta\left(y_{i}, x\right)\right) \notin C, \forall i=1,2, \ldots, p$, which implies that $y_{i}-x+x^{4}<0, \forall i=1,2, \ldots, p$. Summing over $i$ we have $x^{4}<0$, which is a contradiction. However, $h$ is not properly $(C, \eta)$ quasimonotone of the Minty type because for $y_{1}=1, y_{2}=2, y_{3}=3, \lambda_{i}=1 / 3$, for $i=1,2,3$ we have $x=\sum_{i=1}^{3} \lambda_{i} y_{i}=2$ and we note that $h\left(y_{i} ; \eta\left(x, y_{i}\right)\right) \notin-C$ for all $i=1,2,3$.

Example 2.3 Let $K=[0,1]$ and $h: K \times R \rightarrow R^{2}$ be defined as

$$
h(x ; d)= \begin{cases}(0,0) & \text { if } x=0, \\ (d, 0) & \text { if } 0<x \leq 1 .\end{cases}
$$

Let $C=R_{+}^{2}$ and $\eta: K \times K \rightarrow R$ be defined as $\eta(x, y)=y-2 x$. We assert that $h$ is properly $(C, \eta)$-quasimonotone of the Minty type on $K$. On the contrary suppose that there exist $y_{1}, y_{2}, \ldots, y_{p} \in K$ and $\lambda_{i} \geq 0, i=1,2, \ldots, p$ with $\sum_{i=1}^{p} \lambda_{i}=1$ and $x=\sum_{i=1}^{p} \lambda_{i} y_{i}$ such that $h\left(y_{i} ; \eta\left(x, y_{i}\right)\right) \notin-C, \forall i=1,2, \ldots, p$ which implies that $y_{i}-2 x>0, \forall i=1,2, \ldots, p$. Summing over $i$ we have $x<0$ which is a contradiction as $x \in K$. Therefore, $h$ is properly $(C, \eta)$-quasimonotone of the Minty type. It can be seen that $h$ is not properly $(C, \eta)$ quasimonotone of the Stampacchia type on $K$ because for $y_{1}=1 / 2, y_{2}=1, \lambda_{i}=1 / 2$, for $i=1,2$, we have $x=\sum_{i=1}^{2} \lambda_{i} y_{i}=3 / 4$ and $h\left(x ; \eta\left(y_{i}, x\right)\right) \notin C, \forall i=1,2$.

The following proposition is an extension of Lemma 2.1 of [14] and Lemma 2.1 of [16] in the finite dimensional case.

Proposition 2.1 If $h$ is properly $(C, \eta)$-quasimonotone of the Stampacchia type and is also $(C, \eta)$-pseudomonotone on $K$, then $h$ is properly $(C, \eta)$-quasimonotone of the Minty type.

Proof The result follows directly from Definitions 2.1 and 2.2.

The next proposition gives sufficient conditions for the map $h$ to be properly $(C, \eta)$ quasimonotone of the Stampacchia type.

Proposition 2.2 If the following conditions hold:

(i) $h(x ; \eta(x, x)) \in C, \forall x \in K$; 
(ii) the mapping $y \rightarrow h(x ; \eta(y, x))$ is a $*$-quasiconvex on $K$, that is, for all $u \in C^{*}=\left\{u \in R^{m} \mid\langle u, v\rangle \geq 0, \forall v \in C\right\}$ we have

$$
\left\langle u, h\left(x ; \eta\left(t y_{1}+(1-t) y_{2}, x\right)\right)\right\rangle \leq \max \left\{\left\langle u, h\left(x ; \eta\left(y_{1}, x\right)\right)\right\rangle,\left\langle u, h\left(x ; \eta\left(y_{2}, x\right)\right)\right\rangle\right\} ;
$$

then $h$ is properly $(C, \eta)$-quasimonotone of the Stampacchia type on $K$.

Proof Let $y_{1}, y_{2}, \ldots, y_{p} \in K$ and $\lambda_{i} \geq 0, i=1,2, \ldots, p$ with $\sum_{i=1}^{p} \lambda_{i}=1$ and $x=\sum_{i=1}^{p} \lambda_{i} y_{i}$. Then from assumptions (i) and (ii) it follows that for any $u \in C^{*}$

$$
\begin{aligned}
0 & \leq\langle u, h(x ; \eta(x, x))\rangle \\
& \leq \max \left\{\left\langle\left(u, h\left(x ; \eta\left(y_{1}, x\right)\right)\right\rangle,\left\langle u, h\left(x ; \eta\left(y_{2}, x\right)\right)\right\rangle, \ldots,\left\langle u, h\left(x ; \eta\left(y_{p}, x\right)\right)\right\rangle\right\}\right. \\
& =\left\langle u, h\left(x ; \eta\left(y_{i}, x\right)\right)\right\rangle, \quad \text { for some } i \in\{1,2, \ldots, p\} .
\end{aligned}
$$

Then it follows that $h\left(x ; \eta\left(y_{i}, x\right)\right) \in C^{* *}=C$ and hence $h$ is properly $(C, \eta)$-quasimonotone of the Stampacchia type.

Remark 2.3 If $h$ is $(C, \eta)$-pseudomonotone and satisfies the assumptions of Proposition 2.2 then by Proposition $2.1 h$ is properly $(C, \eta)$-quasimonotone of the Minty type on $K$.

Remark 2.4 The conditions of the Proposition 2.1 are not necessary as can be seen from the following example.

Example 2.4 Let $K=R$ and $h: K \times R \rightarrow \bar{R}^{2}$ be defined as $h(x ; d)=(-2 d-1,0)$. Let $C=R_{+}^{2}$ and $\eta: K \times K \rightarrow R$ be defined as $\eta(x, y)=y-x$. Then it is easy to verify that $h$ is properly $(C, \eta)$-quasimonotone of the Minty type on $K$. It can be observed that $h$ is $(C, \eta)$ pseudomonotone on $K$. Here, $h$ is not properly $(C, \eta)$-quasimonotone of the Stampacchia type because for $y_{1}=1 / 2, y_{2}=1, \lambda_{i}=1 / 2$ for $i=1,2$, we have $x=\sum_{i=1}^{2} \lambda_{i} y_{i}=3 / 4$ and $h\left(x ; \eta\left(y_{i}, x\right)\right) \notin C, \forall i=1,2$.

\subsection{Relation with generalized montonicity}

In this section, we discuss the relationship of proper quasimonotonicity with pseudomonotonicity and quasimonotonicity.

Remark 2.5 Unlike the operators wherein every pseudomonotone operator is properly quasimonotone (in the Minty sense) (see [7]) we do not have such a relation for the bifunction $h$ as can be seen from the following examples.

Example 2.5 Let $K=R, C=R_{+}^{2}$ and $h: K \times R \rightarrow R^{2}$ be defined as $h(x ; d)=(d,-d)$. Let $\eta: K \times K \rightarrow R$ be defined as $\eta(x, y)=(x-y)^{2}$. Then we observe that $h$ is $(C, \eta)$ pseudomonotone on $K$ but is not properly $(C, \eta)$-quasimonotone of the Minty type on $K$ because for $y_{1}=1, y_{2}=2, y_{3}=4, \lambda_{i}=1 / 3$, for $i=1,2,3$ we have $x=\sum_{i=1}^{3} \lambda_{i} y_{i}=7 / 3$ and $h\left(y_{i} ; \eta\left(x, y_{i}\right)\right) \notin-C$ for all $i=1,2,3$. Also, $h$ is not properly $(C, \eta)$-quasimonotone of the Stampacchia type as $h\left(x ; \eta\left(y_{i}, x\right)\right) \notin C$, for all $i=1,2,3$. 
In case of an operator $T: X \rightarrow 2^{X^{*}}$, where $X$ is a Banach space and $X^{*}$ is the dual space, it was shown in [7] that if $T$ is properly quasimonotone in the Minty sense then it is quasimonotone. But without any specific assumptions on $h$ and $\eta$ there is no relation between proper $(C, \eta)$-quasimonotonicity of the Minty type and $(C, \eta)$-quasimonotonicity, as the following two examples put in evidence.

Example 2.6 Let $K, C$ and $\eta$ be the same as in the above example. Let $h: K \times R \rightarrow \bar{R}^{2}$ be defined as

$$
h(x ; d)= \begin{cases}(1,1) & \text { if } d=1, \\ (-1,-1) & \text { otherwise }\end{cases}
$$

Then as shown in the above example the bifunction $h$ is not $(C, \eta)$-quasimonotone on $K$ and hence not $(C, \eta)$-pseudomonotone on $K$. We assert that the bifunction $h$ is properly $(C, \eta)$-quasimonotone of the Minty type on $K$. Suppose on the contrary there exist $y_{1}, y_{2}, \ldots, y_{p} \in K$ and $\lambda_{i} \geq 0, i=1,2, \ldots, p$ with $\sum_{i=1}^{p} \lambda_{i}=1$ and $x=\sum_{i=1}^{p} \lambda_{i} y_{i}$ such that $h\left(y_{i} ; \eta\left(x, y_{i}\right)\right) \notin-C, \forall i=1,2, \ldots, p$. This implies that $x+y_{i}=1, \forall i=1,2, \ldots, p$, which cannot be true. But $h$ is not properly $(C, \eta)$-quasimonotone of the Stampacchia type on $K$ because for $y_{1}=1 / 2, y_{2}=1, \lambda_{i}=1 / 2$ for $i=1,2$, we have $x=\sum_{i=1}^{2} \lambda_{i} y_{i}=3 / 4$ and $h\left(x, \eta\left(y_{i}, x\right)\right) \notin C$, $\forall i=1,2$.

Example 2.7 Let $K=[0, \pi / 2] \times[0, \pi / 2], C=R^{2}$ and $h: K \times R^{2} \rightarrow \bar{R}^{2}$ be defined as $h(x ; d)=\left(d_{1} \cos x_{2}, d_{2} \sin x_{1}\right)$, where $x=\left(x_{1}, x_{2}\right)$ and $d=\left(d_{1}, d_{2}\right)$. Let $\eta: K \times K \rightarrow R^{2}$ be defined as $\eta(x, y)=\left(\sin x_{1}-\sin y_{1}, \cos x_{2}-\cos y_{2}\right)$. Then $h$ is $(C, \eta)$-quasimonotone on $K$ but $h$ is not properly $(C, \eta)$-quasimonotone of the Minty type on $K$ because for $y_{1}=(\pi / 6, \pi / 3)$, $y_{2}=(\pi / 9, \pi / 9), y_{3}=(\pi / 3, \pi / 6)$ and $\lambda_{1}=1 / 6, \lambda_{2}=2 / 3, \lambda_{3}=1 / 6$ we have $x=\sum_{i=1}^{3} \lambda_{i} y_{i}=$ $(17 \pi / 108,17 \pi / 108)$ but $h\left(y_{i} ; \eta\left(x, y_{i}\right)\right) \notin-C, \forall i=1,2,3$, and also for these set of points $h\left(x ; \eta\left(y_{i}, x\right)\right) \notin C, \forall i=1,2,3$. Therefore $h$ is not properly $(C, \eta)$-quasimonotone of the Stampacchia type as well. Also, $h$ fails to be $(C, \eta)$-pseudomonotone on $K$ because for $x=(0,0)$ and $y=(\pi / 3, \pi / 4)$ we have $h(x ; \eta(y, x)) \in C$ but $h(y ; \eta(x, y)) \notin-C$.

The following result is an extension of Proposition 1.3 of [18] to the vector case.

Theorem 2.1 Let $h$ be positively homogeneous in the second argument, $\eta$ be linear in the first argument and $\eta(x, x)=0, \forall x \in K$. If $h$ is properly $(C, \eta)$-quasimonotone of the Minty type on $K$ then $h$ is $(C, \eta)$-quasimonotone on $K$.

Proof Let $x, y \in K$ be such that

$$
h(x ; \eta(y, x)) \in \operatorname{int} C .
$$

Let $y_{t}=t x+(1-t) y$, for $t \in(0,1)$. Now since $\eta$ is linear in the first argument and $\eta(x, x)=0, \forall x \in K$, we have $h\left(x ; \eta\left(y_{t}, x\right)\right)=h(x ;(1-t) \eta(y, x))$. By positive homogeneity of $h$ in the second argument it follows that $h\left(x ; \eta\left(y_{t}, x\right)\right) \in \operatorname{int} C$. As $h$ is properly $(C, \eta)$ quasimonotone of the Minty type and $y_{t} \in \operatorname{co}\{x, y\}$ therefore from (1) it follows that $h\left(y ; \eta\left(y_{t}, y\right)\right) \in-C$ that is, $h(y ; t \eta(x, y)) \in-C$. Again invoking positive homogeneity of $h$ in the second argument we get $h(y ; \eta(x, y)) \in-C$. 
Also there does not exist any relationship between proper $(C, \eta)$-quasimonotonicity of the Stampacchia type and $(C, \eta)$-quasimonotonicity of the Minty type.

Example 2.8 Let $K=[0,1]$ and $h: K \times R \rightarrow \bar{R}^{2}$ be defined as

$$
h(x ; d)= \begin{cases}(1,1) & \text { if } d=1 \\ (0,0) & \text { otherwise }\end{cases}
$$

Let $C=R_{+}^{2}$ and $\eta: K \times K \rightarrow R$ be defined as $\eta(x, y)=x+y$. Then for $x=0$ and $y=1, h(x ; \eta(y, x)) \in \operatorname{int} C$ but $h(y ; \eta(x, y)) \notin-C$. Thus, $h$ is not $(C, \eta)$-quasimonotone on $K$. Clearly, $h$ is properly $(C, \eta)$-quasimonotone of the Minty type as well as Stampacchia type on $K$.

The next example is that of a bifunction which is $(C, \eta)$-quasimonotone as well as properly $(C, \eta)$-quasimonotone of the Stampacchia type but not properly $(C, \eta)$ quasimonotone of the Minty type.

Example 2.9 Let $K=R^{2}, C=R_{+}^{2}$ and $h: K \times R^{2} \rightarrow \bar{R}^{2}$ be defined as

$$
h(x ; d)= \begin{cases}\left(1+x_{1}+x_{2}-d_{1}-d_{2}, 0\right) & \text { if } x=(0,1), \\ \left(d_{1}, 1 / 2\right) & \text { if } x=(0,0), \\ \left(0, d_{2}\right) & \text { if } x=(1,0), \\ (0,0) & \text { otherwise }\end{cases}
$$

Let $\eta: K \times K \rightarrow R^{2}$ be defined as $\eta(x ; y)=\left(x_{1}+y_{1}, x_{2}+y_{2}\right)$. Then it can be verified that $h$ is properly $(C, \eta)$-quasimonotone of the Stampacchia type and is also $(C, \eta)$-quasimonotone on $K$. But $h$ is not $(C, \eta)$-pseudomonotone on $K$ because for $x=(0,1)$ and $y=(1,0)$ we have $h(x ; \eta(y, x)) \in C$ but $h(y ; \eta(x, y)) \notin-C$. Also $h$ is not properly $(C, \eta)$-quasimonotone of the Minty type because for $y_{1}=(0,1), y_{2}=(1,0), y_{3}=(0,0)$ and $\lambda_{i}=1 / 3$, for $i=1,2,3$ we have $x=(1 / 3,1 / 3)$ and we note that $h\left(y_{i} ; \eta\left(x, y_{i}\right)\right) \notin-C$ for $i=1,2,3$.

Remark 2.6 The relationship between different classes of bifunctions, studied in this section, is summarized in the following diagram (see Figure 1), where $p m, q m, p q m S$, pqmM denote the class of $(C, \eta)$-pseudomonotone, $(C, \eta)$-quasimonotone, properly $(C, \eta)$-quasimonotone of the Stampacchia type, properly $(C, \eta)$-quasimonotone of the Minty type, respectively.

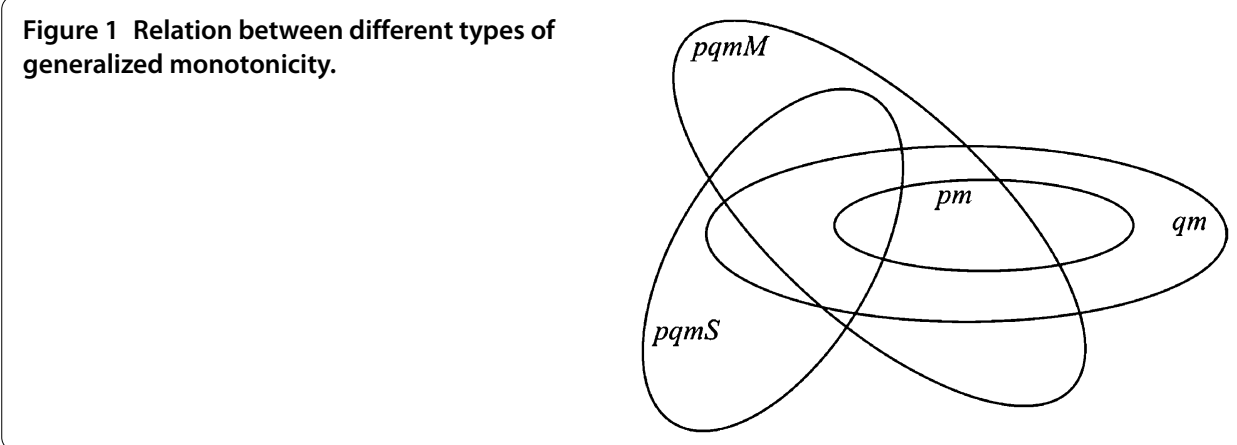




\section{Strong vector variational like inequality problems}

In this section, we consider the following strong vector variational like inequalities of the Stampacchia and the Minty type. The Stampacchia type vector variational like inequality is to find $x \in K$ such that

$$
h(x ; \eta(y, x)) \in C, \quad \forall y \in K,
$$

and the Minty type vector variational like inequality is to find $x \in K$ such that

$$
h(y ; \eta(x, y)) \in-C, \quad \forall y \in K .
$$

Some special cases are as follows:

(i) When $m=1$ and $C=R_{+}$the problem (SVVLI) was studied by Mehta in [21] wherein existence theorems and relation with an optimization problem were studied.

(ii) When $h(x ; \eta(y, x))=\langle T(x), \eta(y, x)\rangle$ where $T: R^{n} \rightarrow R^{m \times n}$ then the problems (SVVLI) and (MVVLI) are the finite dimensional versions of the problems studied by Zhao and Xia [16].

(iii) If $h(x ; \eta(y, x))=\langle T(x), \eta(y, x)\rangle$ and $\eta(x, y)=x-g(y)$ where $T: R^{n} \rightarrow R^{m \times n}$ and $g: K \rightarrow R^{n}$ then the problems (SVVLI) and (MVVLI), respectively, reduce to the problem of finding $x \in K$ such that $\langle T(x), y-g(x)\rangle \in C, \forall y \in K$ and

$$
\langle T(y), x-g(y)\rangle \in-C, \quad \forall y \in K
$$

These are the finite dimensional versions of the strong implicit vector variational inequality problems that were considered by Fang and Huang [14].

(iv) If $h(x ; \eta(y, x))=\langle T(x), \eta(y, x)\rangle$ where $\eta(x, y)=x-y$ then the problems (SVVLI) and (MVVLI), respectively, reduce to the problems of finding $x \in K$ such that $\langle T(x), y-x\rangle \in C, \forall y \in K$ and

$$
\langle T(y), x-y\rangle \in-C, \quad \forall y \in K
$$

which were the problems studied by Komlosi [15] and referred to as 'neutral' Stampacchia and Minty vector variational inequalities, respectively.

Definition 3.1 The vector bifunction $h$ is said to be $(C, \eta)$-subodd on $K$ if for each $x, y \in K$ we have $h(x ; \eta(y, x))+h(x ; \eta(x, y)) \in C$.

Remark 3.1 If $\eta(x, y)+\eta(y, x)=0$, then the above definition reduces to the definition of $C$-subodd given by Lalitha and Mehta [9].

The next theorem is a generalization of the famous Minty Lemma [22].

Theorem 3.1 If the bifunctions $h$ and $\eta$ satisfy the following conditions:

(i) $h$ is $(C, \eta)$-pseudomonotone on $K$;

(ii) for each $d \in R^{n}$, the mapping $x \rightarrow h(x ; d)$ is hemicontinuous, that is, for any $x, y \in R^{n}, \lim _{t \rightarrow 0^{+}} h(x+t(y-x) ; d)=h(x ; d)$;

(iii) $\eta$ is affine in the second argument and $\eta(x, x)=0, \forall x \in K$; 
(iv) $h$ is $(C, \eta)$-subodd on $K$;

(v) $h$ is positively homogeneous in the second argument;

then the problems (SVVLI) and (MVVLI) are equivalent.

Proof As $h$ is $(C, \eta)$-pseudomonotone on $K$ therefore it follows that every solution of (SVVLI) is also a solution of (MVVLI). Conversely, let $x \in K$ be a solution of (MVVLI) then we have

$$
h(y ; \eta(x, y)) \in-C, \quad \forall y \in K .
$$

Let $y \in K$ and let $z_{t}=x+t(y-x)$, for $t \in(0,1)$. Then it follows that $h\left(z_{t} ; \eta\left(x, z_{t}\right)\right) \in$ $-C$, and since $\eta$ is affine in the second argument and $\eta(x, x)=0, \forall x \in K$, it follows that $h\left(z_{t} ; t \eta(x, y)\right) \in-C$. Using assumption (v) we get $h\left(z_{t} ; \eta(x, y)\right) \in-C$, and by assumption (ii) on letting $t \rightarrow 0^{+}$, we have $h(x ; \eta(x, y)) \in-C$. Then $(C, \eta)$-suboddness of $h$ yields $h(x ; \eta(y, x)) \in C$, that is, $x$ solves (SVVLI).

We now give an example to illustrate the above result.

Example 3.1 Let $K=R^{2}, C=R_{+}^{2}$, and let $h: K \times R^{2} \rightarrow \bar{R}^{2}$ be defined as

$$
h(x ; d)= \begin{cases}\left(x_{1} d_{1}, x_{2} d_{1}^{3} / d_{2}^{2}\right) & \text { if } d_{2} \neq 0 \\ (0,0) & \text { if } d_{2}=0\end{cases}
$$

Let $\eta: K \times K \rightarrow R^{2}$ be defined as $\eta(x, y)=\left(x_{1}-y_{1}, y_{2}-x_{2}\right)$. Then we note that $h$ is a positively homogeneous function in the second argument and $h$ is $(C, \eta)$-subodd on $K$ as well. Clearly, $h$ is $(C, \eta)$-pseudomonotone on $K$. So all the conditions of the above theorem are satisfied and it can be seen that $\left\{\left(0, x_{2}\right) \mid x_{2} \geq 0\right\}$ is the solution set of the problems (SVVLI) and (MVVLI).

We now give an existence theorem for the problem (SVVLI) using the KKM lemma.

Definition 3.2 A point to set map $F: X \subseteq R^{n} \rightarrow 2^{X}$ is called a KKM map, if for every finite subset $\left\{x_{1}, x_{2}, \ldots, x_{p}\right\}$ of $X, \operatorname{co}\left\{x_{1}, x_{2}, \ldots, x_{p}\right\} \subseteq \bigcup_{i=1}^{p} F\left(x_{i}\right)$.

Lemma 3.1 ([23]) Let $X$ be a nonempty, convex subset of $R^{n}$. Let $F: X \rightarrow 2^{X}$ be a KKMmap such that for each $x \in X, F(x)$ is closed and is compact for at least one $x \in X$ then $\bigcap_{x \in X} F(x) \neq \varphi$.

The following two theorems are extensions of Theorems 2.1 and 2.2 of [14] in the finite dimensional case.

Theorem 3.2 Let $K$ be a nonempty compact convex subset of $R^{n}$ If the bifunctions $h$ and $\eta$ satisfy the following conditions:

(i) for each $y \in K$, the mapping $x \rightarrow h(x ; \eta(y, x))$ is continuous;

(ii) $h(x ; \eta(x, x)) \in C, \forall x \in K$;

(iii) $h$ is properly $(C, \eta)$-quasimonotone of the Stampacchia type on $K$; then the problem (SVVLI) is solvable. 
Proof Define the set-valued mapping $F_{1}: K \rightarrow 2^{K}$ as

$$
F_{1}(y)=\{x \in K \mid h(x ; \eta(y, x)) \in C\} .
$$

Then by assumption (ii) it follows that for each $y \in K, y \in F_{1}(y)$ and hence $F_{1}(y) \neq \phi$. Next we prove that $F_{1}$ is a KKM-map. On the contrary suppose that there exists $x_{1}, x_{2}, \ldots, x_{p} \in$ $K$ and $x \in \operatorname{co}\left\{x_{1}, x_{2}, \ldots, x_{p}\right\}$ such that $x \notin \bigcup_{i=1}^{p} F_{1}\left(x_{i}\right)$. This implies that $h\left(x ; \eta\left(x_{i}, x\right)\right) \notin C$, $\forall i=1,2, \ldots, p$ which contradicts assumption (iii). Thus, $F_{1}$ is a KKM-map. Assumption (i) ensures that $F_{1}(y)$ is a closed subset of $K$ and since $K$ is compact it follows that $F_{1}(y)$ is compact, for each $y \in K$. Hence by Lemma 3.1 we have $\bigcap_{y \in K} F_{1}(y) \neq \varphi$ that is, the problem (SVVLI) is solvable.

We now establish an existence theorem for (MVVLI) using the proper $(C, \eta)$-quasimonotonicity of $h$ of the Minty type.

Theorem 3.3 Let $K$ be a nonempty compact convex subset of $R^{n}$ If the bifunctions $h$ and $\eta$ satisfy the following conditions:

(i) for each $y \in K$, the mapping $x \rightarrow h(y ; \eta(x, y))$ is continuous;

(ii) $h(x ; \eta(x, x)) \in-C, \forall x \in K$;

(iii) $h$ is properly $(C, \eta)$-quasimonotone of the Minty type on $K$;

then the problem (MVVLI) is solvable.

Proof Define the set-valued mapping $F_{2}: K \rightarrow 2^{K}$ as

$$
F_{2}(y)=\{x \in K \mid h(y ; \eta(x, y)) \in-C\} .
$$

Proceeding on the lines of the above theorem it can be shown that $\bigcap_{y \in K} F_{2}(y) \neq \varphi$, that is, (MVVLI) has a solution.

\section{Gap functions}

It is well known that the concept of gap functions is very important for the study of vector variational inequality problems. Until now, two methods have been used for introducing gap functions for vector variational inequality problems: one is of single-valued type and the other is of vector-valued type. But single-valued gap functions are computationally more tractable. Yang and Yao [24] defined gap functions for vector variational inequality problems as real-valued functions, whereas Chen et al. [25] defined gap functions for vector variational inequality problems as set-valued maps.

We now propose two gap functions for a Stampacchia type vector variational like inequality problem (SVVLI).

Definition 4.1 A function $g: K \rightarrow R \cup\{-\infty\}$ is said to be a gap function for the problem (SVVLI) if

(i) $g(x) \leq 0, \forall x \in K$;

(ii) $g(\hat{x})=0$ if and only if $\hat{x}$ solves (SVVLI).

Define $\Phi: K \rightarrow R \cup\{-\infty\}$ as

$$
\Phi(x)=\inf _{y \in K} \inf _{u \in B^{*}}\langle u, h(x ; \eta(y, x))\rangle,
$$

where $B^{*}=\left\{u \in C^{*} \mid\|u\|=1\right\}$. If $C$ is a closed convex cone then $C^{* *}=C$. 
Theorem 4.1 The function $\Phi$ is a gap function for the problem (SVVLI) provided $h(x ; \eta(x, x))=0, \forall x \in K$.

Proof (i) For any $x \in K, \Phi(x) \leq \inf _{u \in B^{*}}\langle u, h(x ; \eta(y, x))\rangle=0, \forall y \in K$. In particular, for $y=x$ we have $\Phi(x) \leq \inf _{u \in B^{*}}\langle u, h(x ; \eta(x, x))\rangle=0$.

(ii) Now $\Phi(\hat{x})=0$ is equivalent to the fact that $\inf _{u \in B^{*}}\langle u, h(\hat{x} ; \eta(y, \hat{x}))\rangle \geq 0, \forall y \in K$ which is further equivalent to the fact that $\langle u, h(\hat{x} ; \eta(y, \hat{x}))\rangle \geq 0, \forall y \in K, \forall u \in C^{*}$ which holds if and only if $h(\hat{x} ; \eta(y, \hat{x})) \in C^{* *}, \forall y \in K$, that is, $\hat{x}$ solves (SVVLI).

We now propose another gap function for (SVVLI) when $C=R_{+}^{m}$. Let

$$
h(x ; \eta(y, x))=\left(h_{1}(x ; \eta(y, x)), h_{2}(x ; \eta(y, x)), \ldots, h_{m}(x ; \eta(y, x))\right) .
$$

Define $\Phi^{\prime}: K \rightarrow R \cup\{-\infty\}$ as

$$
\Phi^{\prime}(x)=\inf _{y \in K} \min _{1 \leq i \leq m} h_{i}(x ; \eta(y, x)) .
$$

Theorem 4.2 The function $\Phi^{\prime}$ is a gap function for the problem (SVVLI) when $C=R_{+}^{m}$ provided $h(x ; \eta(x, x))=0, \forall x \in K$.

Proof For any $x \in K, \Phi^{\prime}(x) \leq \min _{1 \leq i \leq m} h_{i}(x ; \eta(y, x)), \forall y \in K$. In particular, for $y=x$ we have $\Phi^{\prime}(x) \leq \min _{1 \leq i \leq m} h_{i}(x ; \eta(x, x))=0$. Now $\Phi^{\prime}(\hat{x})=0$ is equivalent to the fact that $\min _{1 \leq i \leq m} h_{i}(\hat{x} ; \eta(y, \hat{x})) \geq 0, \forall y \in K$ which is further equivalent to the fact that $h(\hat{x} ; \eta(y, \hat{x})) \in$ $R_{+}^{m}, \forall y \in K$ that is, $\hat{x}$ solves (SVVLI).

We now give an example to show that the solution of the problem (SVVLI) can easily be computed using the gap function.

Example 4.1 Let $K=[-1,2] \times[0,1], C=R_{+}^{2}$ and $h: K \times R^{2} \rightarrow \bar{R}^{2}$ be defined as $h(x ; d)=$ $\left(h_{1}(x ; d) h_{2}(x ; d)\right)=\left(x_{1}+x_{2}-d_{1}, d_{2}-x_{1}-x_{2}\right)$, where $x=\left(x_{1}, x_{2}\right)$ and $d=\left(d_{1}, d_{2}\right)$. Let $\eta$ : $K \times K \rightarrow R^{2}$ be defined as $\eta(x, y)=\left(x_{1}+y_{2}, x_{2}+y_{1}\right)$, where $y=\left(y_{1}, y_{2}\right)$. Then $h(x ; \eta(x, x))=0$, $\forall x \in K$ and $\hat{x}=(2,0)$ is the only solution of (SVVLI). By taking $\|u\|=\left|u_{1}\right|+\left|u_{2}\right|$ the gap function $\Phi$ is given as

$$
\begin{aligned}
\Phi(x) & =\inf _{y \in K} \inf _{u \in B^{*}}\langle u, h(x ; \eta(y, x))\rangle \\
& =\min _{y \in K} \inf _{u \in B^{*}}\left\{u_{1}\left(x_{1}-y_{1}\right)+\left(u_{2}\right)\left(y_{2}-x_{2}\right)\right\} \\
& =\min _{y \in K} \inf _{0 \leq u_{1} \leq 1}\left\{u_{1}\left(x_{1}-y_{1}\right)+\left(1-u_{1}\right)\left(y_{2}-x_{2}\right)\right\} .
\end{aligned}
$$

Since

$$
\inf _{0 \leq u_{1} \leq 1}\left\{u_{1}\left(x_{1}-y_{1}\right)+\left(1-u_{1}\right)\left(y_{2}-x_{2}\right)\right\}= \begin{cases}y_{2}-x_{2} & \text { if } x_{1}+x_{2} \geq y_{1}+y_{2} \\ x_{1}-y_{1} & \text { if } x_{1}+x_{2}<y_{1}+y_{2}\end{cases}
$$


we have $\Phi(x)=\min \left\{x_{1}-2,-x_{2}\right\}$. Clearly $\Phi(x) \leq 0, \forall x \in K$ and $\Phi(\hat{x})=0$ for $\hat{x}=(2,0)$. Also $\Phi^{\prime}$ is given as

$$
\begin{aligned}
\Phi^{\prime}(x) & =\inf _{y \in K} \min _{1 \leq i \leq m} h_{i}(x ; \eta(y, x)) \\
& =\inf _{y \in K} \min \left\{x_{1}-y_{1}, y_{2}-x_{2}\right\} \\
& =\min \left\{x_{1}-2,-x_{2}\right\} .
\end{aligned}
$$

So $\Phi^{\prime}(x) \leq 0, \forall x \in K$ and $\Phi^{\prime}(\hat{x})=0$ for $\hat{x}=(2,0)$.

Remark 4.1 If in the above example we define $\eta$ as $\eta(x, y)=\left(x_{1}+y_{2}, x_{1}+x_{2}\right)$, then the gap function $\Phi^{\prime}$ for the problem (SVVLI) is given as

$$
\Phi^{\prime}(x)=\min \left\{x_{1}-2,-x_{1}-x_{2}-1\right\}
$$

We observe that $\Phi^{\prime}(x) \leq 0, \forall x \in K$ but $\Phi^{\prime}(x) \neq 0$ for any $x \in K$. Thus, the problem (SVVLI) does not have a solution.

It is also possible to define gap functions as defined above for Minty type vector variational like inequality problem.

Definition 4.2 A function $g: K \rightarrow R \cup\{\infty\}$ is said to be a gap function for the problem (MVVLI) if

(i) $g(x) \geq 0, \forall x \in K$;

(ii) $g(\hat{x})=0$ if and only if $\hat{x}$ solves (MVVLI).

The first gap function for the problem (MVVLI) $\psi: K \rightarrow R \cup\{\infty\}$ is defined as

$$
\psi(x)=\sup _{y \in K} \sup _{u \in C^{*}}\langle u, h(x ; \eta(y, x))\rangle .
$$

The following theorem can be established on the lines of Theorem 4.1.

Theorem 4.3 The function $\psi$ is a gap function for the problem (MVVLI) provided $h(x ; \eta(x, x))=0, \forall x \in K$.

We now propose a second gap function for (MVVLI) when $C=R_{+}^{m}$.

Define $\psi^{\prime}: K \rightarrow R \cup\{\infty\}$ as

$$
\psi^{\prime}(x)=\sup _{y \in K} \max _{1 \leq i \leq m} h_{i}(x ; \eta(y, x)) .
$$

The following theorem can be established along the lines of Theorem 4.2.

Theorem 4.4 The function $\psi$ ' is a gap function for the problem (MVVLI) when $C=R_{+}^{m}$ provided $h(x ; \eta(x, x))=0, \forall x \in K$.

Remark 4.2 For the bifunction $h$ considered in Example 4.1 the solution for the Minty type vector variation like inequality problem (MVVLI) is also $\hat{x}=(2,0)$. It is noteworthy that all the conditions of Theorem 3.1 are satisfied and hence the problems (SVVLI) 
and (MVVLI) are equivalent. The gap functions can also be evaluated for the problem (MVVLI) along lines similar to the problem (SVVLI).

\section{Competing interests}

The authors declare that they have no competing interests.

\section{Authors' contributions}

Both the authors have contributed equally in the development of this study. Both authors have read and approved the final manuscript.

\section{Acknowledgements}

The authors are grateful to Dr. C.S. Lalitha, Associate Professor, Department of Mathematics, University of Delhi South

Campus, New Delhi and the referees for their valuable inputs that helped in improving the quality of the paper.

Received: 6 April 2013 Accepted: 29 January 2014 Published: 07 Apr 2014

\section{References}

1. Giannessi, F: Theorems of alternative, quadratic programs and complementarity problems. In: Cottle, RW, Gianessi, F Lions, JL (eds.) Variational Inequality and Complementarity Problems, pp. 151-186. Wiley, Chichester (1980)

2. Ansari, QH: A note on generalized vector variational like-inequalities. Optimization 41, 197-205 (1997)

3. Garzon, GR, Gomez, RO, Lizano, AR: Relationships between vector variational-like inequality and optimization problems. Eur. J. Oper. Res. 157, 113-119 (2004)

4. Al-Homidan, S, Ansari, QH, Yao, JC: Non smooth invexities, invariant monotonicities and non smooth vector variational-like inequalities with applications to vector optimization. In: Ansari, QH, Yao, JC (eds.) Recent Developments in Vector Optimization. Springer, Berlin (2012)

5. Lee, BS, Lee, SJ: Vector variational type inequalities for set valued mappings. Appl. Math. Lett. 13, 57-62 (2000)

6. Li, J, He, ZQ: Gap functions and existence of solutions to generalized vector variational inequalities. Appl. Math. Lett $18,989-1000(2005)$

7. Daniilidis, A, Hadjisavvas, N: On the subdifferential of quasiconvex and pseudoconvex functions and cyclic monotonicity. J. Math. Anal. Appl. 237, 30-42 (1999)

8. John, R: A note on Minty variational inequalities and generalized monotonicity. In: Generalized Convexity and Generalized Monotonicity (Karlovarsi, 1999). Lecture Notes in Economics and Mathematical Systems, vol. 502, pp. 240-246. Springer, Berlin (2001)

9. Lalitha, CS, Mehta, M: Vector variational inequalities with cone-pseudomonotone bifunctions. Optimization 54 327-338 (2005)

10. Lalitha, CS, Mehta, M: On vector variational inequalities problem in terms of bifunctions. Aust. J. Math. Anal. Appl. 3 , $11(2006)$

11. Ansari, QH, Lalitha, CS, Mehta, M: Generalized Convexity, Non Smooth Variational Inequalities and Non Smooth Optimization. CRC Press, Boca Raton (2014)

12. Aussel, D, Luc, DT: Existence conditions in general quasimonotone variational inequalities. Bull. Aust. Math. Soc. 71, 285-303 (2005)

13. Fang, YP, Huang, NJ: Variational like inequalities with generalized monotone mappings in Banach spaces. J. Optim. Theory Appl. 118, 327-338 (2003)

14. Fang, YP, Huang, NJ: Existence results for systems of strong implicit vector variational inequalities. Acta Math. Hung 103, 265-277 (2004)

15. Komlosi, S: On the Stampacchia and Minty variational inequalities. In: Giorgi, G, Rossi, F (eds.) Generalized Convexity and Optimization for Economic and Financial Decisions, pp. 231-260. Pitagora Editrice, Bologna (1999)

16. Zhao, Y, Xia, Z: Existence results for systems of vector variational like inequalities. Nonlinear Anal., Real World Appl. 8 1370-1378 (2005)

17. Zheng, F: Vector variational inequalities with semi-monotone operators. J. Glob. Optim. 32, 633-642 (2005)

18. Bianchi, M, Hadjisavvas, N, Schaible, S: Vector equilibrium problems with generalized monotone bifunctions. J. Optim Theory Appl. 92, 527-542 (1997)

19. Zhou, JX, Chen, G: Diagonal convexity conditions for problems in convex analysis and quasi-variational inequalities. J. Math. Anal. Appl. 132, 213-225 (1988)

20. Bianchi, M, Pini, R: A note on equilibrium problem with properly quasimonotone bifunctions. J. Glob. Optim. 20, 67-76 (2001)

21. Mehta, M: Existence of solution for a generalized variational like inequality problems in terms of bifunctions. Opsearch 44, 41-50 (2007)

22. Minty, GJ: Monotone (nonlinear) operators in Hilbert space. Duke Math. J. 29, 341-346 (1962)

23. Lassonde, M: On the use of KKM multifunction in fixed point theory and related topics. J. Math. Anal. Appl. 97 151-201 (1983)

24. Yang, XQ, Yao, JC: Gap functions and existence of solutions to set valued variational inequalities. J. Optim. Theory Appl. 115, 407-417 (2002)

25. Chen, GY, Goh, CJ, Yang, XQ: Existence of a solution for generalized vector variational inequalities. Optimization 50 , $1-15(2001)$

10.1186/1029-242X-2014-142

Cite this article as: Mehta and Chaudhary: Strong vector variational like inequality problems with properly quasimonotone bifunctions. Journal of Inequalities and Applications 2014, 2014:142 